\title{
SILICON MICROMACHINING FOR HIGH PERFORMANCE PASSIVE STRUCTURES AT W BAND
}

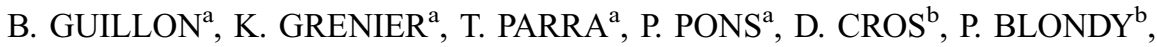 \\ J. GRAFFEUIL ${ }^{\mathrm{a}}$, J. L. CAZAUX ${ }^{\mathrm{c}}$ and R. PLANA ${ }^{\mathrm{a}, *}$ \\ ${ }^{\mathrm{a}}$ LAAS-CNRS $7 \mathrm{Av} d u$ Colonel Roche 31077 Toulouse Cedex04 France; ${ }^{\mathrm{b}}$ IRCOM 123 Av Albert \\ Thomas, 87000 Limoges Cedex France; ${ }^{\mathrm{c}}$ Alcatel Space Industry 26 Av J.F. Champollion 31037 \\ Toulouse France
}

(Received 6 November 2001; In final form 3 December 2001)

\begin{abstract}
This paper presents a micromachined technology allowing the realization of very high aspect ratio millimeter-wave circuits. Appropriate 3D electromagnetic simulations based on the finite element method have been implemented to design the circuits. Coplanar transmission lines featuring loss level in the $2 \mathrm{~dB}$ range up to $105 \mathrm{GHz}$ have been realized. An original silicon micromachined cavity using the whispering gallery modes properties has been realized achieving a quality factor close to 10,000 range at $95 \mathrm{GHz}$.
\end{abstract}

Keywords: Micromachined; Silicon; Whispering gallery mode; 3D FEM simulation; Millimeter-wave

\section{INTRODUCTION}

The future "Multimedia Century" will turn in a dramatic change of the requirements of the microwave and millimeter-wave modules. First of all, the Semiconductor Industrial Association (SIA) roadmap indicates that the allocated frequencies will cover a frequency range from $1 \mathrm{GHz}$ to $77 \mathrm{GHz}$ meaning that there will be a need for circuits featuring very broad band capabilities. Secondly, the circuits should feature high performances in terms of noise, power capabilities, consumption, linearity, reliability, cost and integration. These new trends have stimulated the emergence of silicon technologies. Concerning the active devices, the heterostructure concept has led to the realization of millimeter wave devices. Concerning the passive elements, the situation was more complex and a lot of effort has been invested to improve their quality factor. Among these, one of the most important was the attractive development of silicon micromachining processes. Previous work by L. Katehi and G. Rebeiz at the Michigan University has demonstrated very impressive results [1]. The work presented in this paper concerns a micromachined coplanar technology allowing the realization of very high Q millimeter wave cavities. In the first part we will present briefly the technological process we have developed. The second part will be dedicated to the design of the millimeter

\footnotetext{
* Corresponding author. E-mail: plana@laas.fr
} 
wave structures. The results that have been obtained will be presented in the section III. Finally, conclusions will be outlined in the last section.

\section{TECHNOLOGICAL PROCESS}

The technological process we will present concerns the realization of suspended microwave devices on a thin dielectric membrane. The first step of the process is devoted to the membrane mechanical properties, which must be optimized with respect to areas varying from a few $\mathrm{mm}^{2}$ to several tens of $\mathrm{mm}^{2}$ in order to meet the millimeter-wave range requirements. For mechanical strength reasons, these membranes must feature both a low level of stress and an appropriate thickness typically greater than $1 \mu \mathrm{m}$. The mechanical strength of a layer depends on the intrinsic stress within the layer, which must be lower than $1 \mathrm{GPa}$. Another source of stress is related to the shear stress at the interface between the layer and the host substrate. This interface stress is driven by both, the thickness of the layer and by the intrinsic stress of the two layers. It is very difficult to realize thick monolithic dielectric membrane exhibiting a low level of stress. To overcome this problem, we have developed a composite membrane using two dielectric layers. The first layer elaborated from the silicon substrate consists in a thermal oxide. The main source of stress in this kind of oxide comes from the difference in the thermal expansion coefficients between the silicon and the oxide. At $1150^{\circ} \mathrm{C}$, the oxide layer exhibits a compressive stress of about $300 \mathrm{MPa}$. The second layer consists in a silicon nitride layer realized by low pressure decomposition ( $300 \mathrm{mTorr}$ ) at $750{ }^{\circ} \mathrm{C}$ of Silane $\left(\mathrm{SiH}_{4}\right)$ and Ammonia $\left(\mathrm{NH}_{3}\right)$. The intrinsic stress of the silicon nitride layer depends on the gas ratio $r=\mathrm{NH}_{3} / \mathrm{SiH}_{4}$. An optimal working point is achieved for a value of $r$ close to 0.8 . In this case, the stress of the composite dielectric membrane features a slightly tensile behavior in order to prevent its buckling during its releasing from the silicon substrate. The buckling behavior would affect the spatial geometry of the structure resulting to a change in the electrical properties of the device as well as a degradation of the reliability of the sample. The composite membrane features $800 \mathrm{~nm}$ thick silicon oxide layer and $600 \mathrm{~nm}$ thick silicon nitride layer. The resulting membrane exhibits an overall tensile stress in the $100 \mathrm{MPa}$ range. Reliability tests have been carried out through pressure steps and thermal cycles on membrane featuring areas of $4 \mathrm{~mm}^{2}, 8 \mathrm{~mm}^{2}$ and $12 \mathrm{~mm}^{2}$. We have observed no failure with pressure steps up to 1 bar and several hundred thermal cycles ranging from $-30{ }^{\circ} \mathrm{C}$ to $150^{\circ} \mathrm{C}$. Concerning the microwave section, we have developed a technological process allowing an accurate control of the circuit size and a minimization of the ohmic losses [2]. The process derives from the well known LIGA one [3,4]. The electroplated gold conductors are realized within a photoresist mould achieved from conventional UV equipment. This technique allows to realize very thick metallization in the $10 \mu \mathrm{m}$ range with a very small roughness of $15 \mathrm{~nm}$. The gold resistivity is stabilized around $2.4 \mu \Omega \mathrm{cm}$ through an annealing process performed at $200{ }^{\circ} \mathrm{C}$ under a $\mathrm{N}_{2}$ ambient. Finally, the membrane is released from a back side $\mathrm{KOH}$ etching. The different steps of the technological process are described in Figure 1. More details on the technological process can be found in [5]. The next section will present the design of the micromachined millimeter wave devices.

\section{DESIGN OF MICROMACHINED DEVICES}

A micromachined device is actually a 3D device due to the anisotropic etching properties of silicon. This implies a characteristic impedance mismatch, which is fixed through a tapered 
Electrolytic deposition

(1)
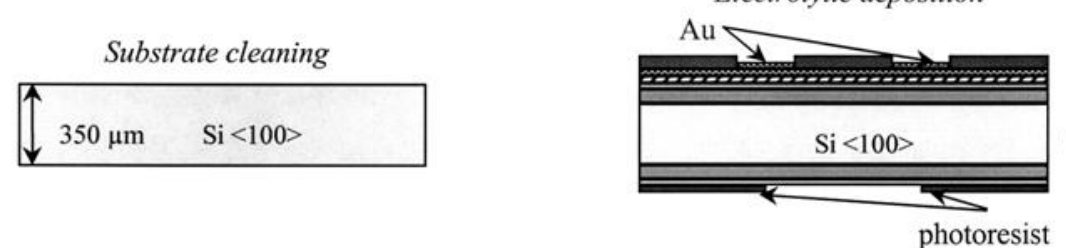

(2)

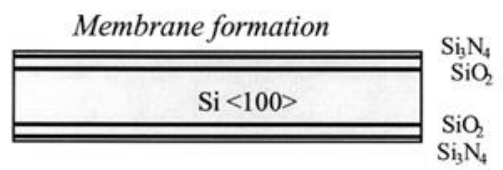

(3)

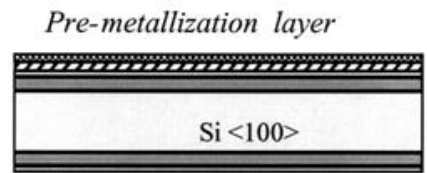

\section{$\mathrm{Au}$}

Photo resist mould realization
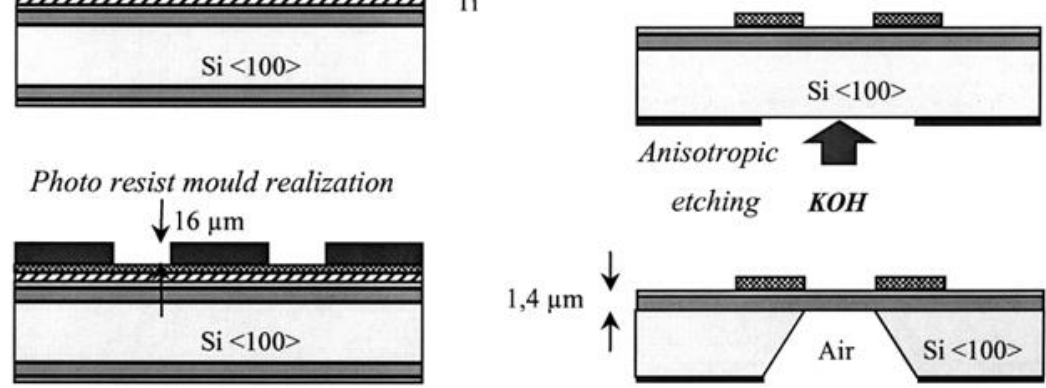

FIGURE 1 Technological process description.

section. We have developed a specific meshing within a 3D Finite Element Method (FEM) based software to design the tapered section as reported in Figure 2. For the membrane section, 2.5D moment based method software has been used. Due to the material discontinuity, a $50 \Omega$ characteristic impedance on the bulk silicon will translate into $125 \Omega$ characteristic

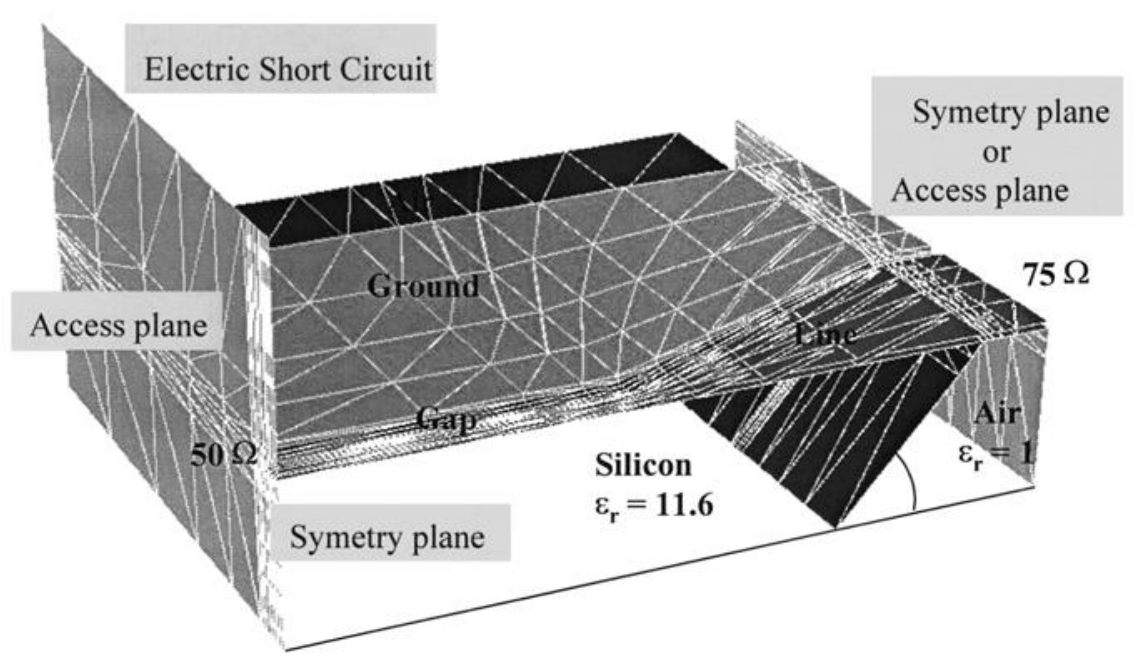

FIGURE 2 3D meshing of a quarter access of a micromachined coplanar line. 
impedance on the membrane section assuming the same geometry. We have designed a coplanar transmission line featuring a low loss tapered section and $6 \mu \mathrm{m}$ metallization thickness in order to decrease the ohmic losses [6]. The membrane section features a $75 \Omega$ characteristic impedance to meet some technological rules associated with the slot width (i.e. a $50 \Omega$ line will lead to narrow slot width which could turn in some reliability problems) and the access part features a $50 \Omega$ characteristic impedance to fit with the measurement test set. Figure 3 is showing the topology of the micromachined coplanar wave guide (MCPW). We have represented in Figure 4 the frequency evolution of the reflection and transmission coefficients of the micromachined transmission line from $1 \mathrm{GHz}$ to $67 \mathrm{GHz}$ as well as the theoretical predictions. We can observe that the transmission line exhibits very attractive performances since we report transmission loss level lower than $1 \mathrm{~dB}$ at $67 \mathrm{GHz}$ and reflection coefficient better than $10 \mathrm{~dB}$. The second point, which can be discussed concerns the good agreement obtained between the data and the theoretical computations achieved through full wave electromagnetic analysis. The slight discrepancy observed on the S11 evolutions is related to the fact that the $6 \mu \mathrm{m}$ metallization thickness turns to a small change in the characteristic impedance value that has not been taken into account during the electromagnetic simulation (i.e. we do not have meshed the metallization). In order to validate this assumption, we have realized CPW transmission featuring metallization thickness of $2 \mu \mathrm{m}$ and the agreement between measured and calculated data were better.

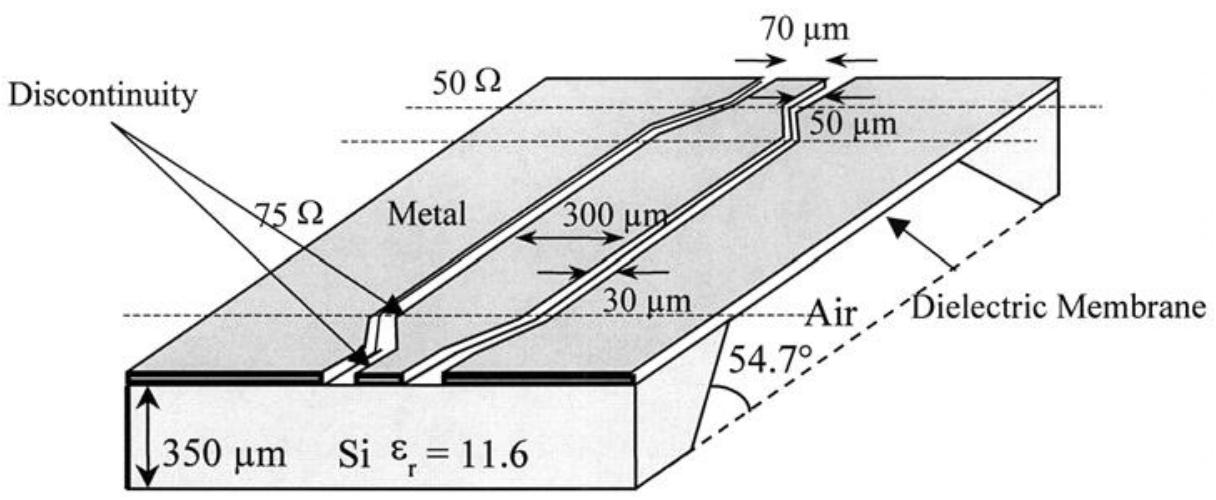

FIGURE 3 Schema of the MCPW transmission line.
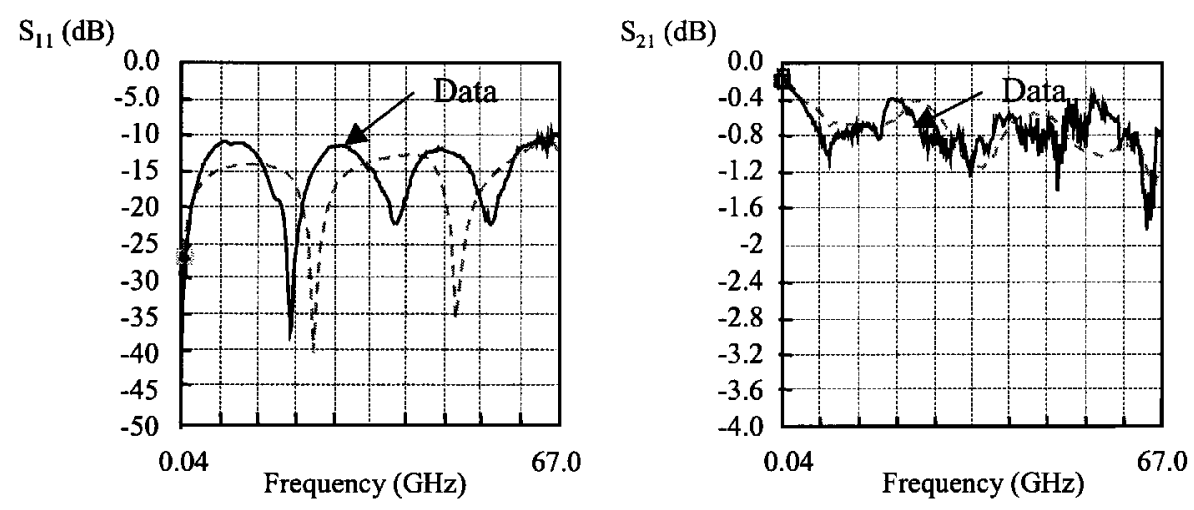

FIGURE 4 Theoretical (dotted line) and experimental (full line) scattering parameters of a $6 \mathrm{~mm}$ length micromachined coplanar wave guide transmission line. 
We have carried out additional work in order to find out about the loss origin and we found that they are almost located in the access of the device due to a MIS structure (Metal-Membrane-Silicon). Similar results have been already reported by Reyes et al. [7]. In order to confirm this behavior, we have biased the transmission line between the top and the substrate and following the DC bias sign, we have got improvement or degradation of the microwave properties of the devices confirming the existence of a depleted or accumulation region under the membrane. Some authors have proposed some solutions to overcome this problem. The first solution consists to remove the dielectric in the slots [8] that will turn to an improvement of the loss level. Recently Ponchak [9] demonstrated that the losses were generated mostly by the $\mathrm{Si}_{3} \mathrm{~N}_{4}$ layer and he proposed to use only an oxide layer realized either by PECVD process or by thermal process. Finally, another solution has been proposed by Gamble et al. [10] consisting to insert a polycristalline silicon layer between the silicon oxide and the high resistivity silicon substrate. This layer features a lot of traps and interface states which will trap the free careers that have been created at the oxide silicon interface. We believe that it will be a successful way to overcome the problems associated with the MIS structure, but we think that alternative solutions could be used like removing the dielectric in the access path (that will involve only an additional mask level) or like using polyimide or BenzoCyclo-Butene layer as a membrane. The second demonstrator, we will present deals with a silicon micromachined microcavity for millimeter-wave range. The first point deals with the kind of devices we have to design in order to get a very high quality coefficient. Some results of the literature have already demonstrated quality factor in the 500 range at both $10 \mathrm{GHz}$ and $60 \mathrm{GHz}[11,12]$ but they concerns cavity acting on conventional modes. We chose to design a microcavity acting on its whispering gallery modes since they feature numerous advantages for millimeter-wave range as: higher quality factor due to a greater Electromagnetic energy confinement inside the resonator, a higher size of the resonator which is important to guarantee a good process repeatability [13]. We have designed a micromachined cavity based on an appropriate coupling between a Silicon Dielectric Resonator and two micromachined coplanar transmission lines at millimeter wave frequency $(95 \mathrm{GHz})$ as reported in Figure 5. The structure consists in a transmission cavity between the accesses I and II due to the propagating character of the whispering gallery modes (WG). Due to the electromagnetic field distribution in the Coplanar Wave Guide (CPW) structure, the coupling is achieved through the magnetic field in the CPW devices and the transversal magnetic field of the Dielectric resonator (DR) exciting their WGE modes family. The resonator is made of a silicon substrate featuring very high resistivity $(8 \mathrm{k} \Omega \mathrm{cm})$. The size of the resonator and the resonant frequency have been obtained through $2 \mathrm{D}$ free oscillations full wave analysis including losses from which we have got a complex frequency $f_{0}=\alpha_{0}+j \beta_{0}$ turning into an unloaded quality factor defined by the following expression:

$$
Q_{0}=\frac{\alpha_{0}}{2 \beta_{0}}
$$
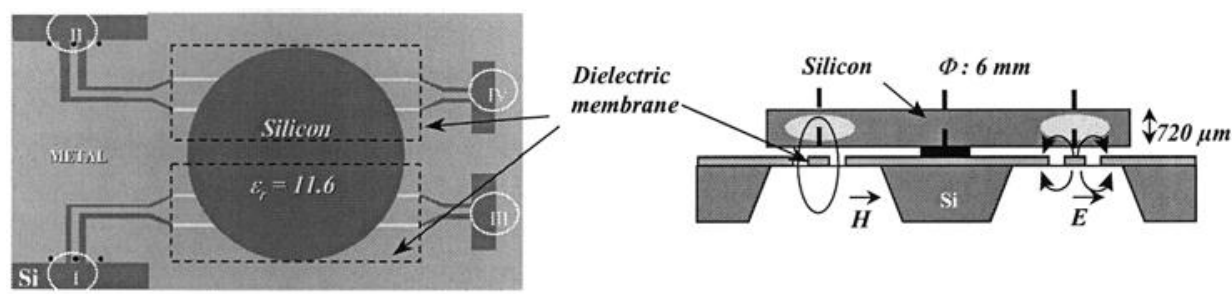

FIGURE 5 Topology of the silicon micromachined cavity in the $95 \mathrm{GHz}$ range. 
The cavity works like a directive filter as reported in Figure 6 and the quality factor of the overall structure is given by the following expression:

$$
1 / Q_{L}=1 / Q_{0}+1 / Q_{e_{1}}+1 / Q_{e_{2}}
$$

where $Q_{e_{1}}$ and $Q_{e_{2}}$ represent the quality factor associated with the input and output circuit respectively

Actually, the structure is fully symmetric and $Q_{e_{1}}=Q_{e_{2}}$

$$
\left|S_{21}\right|_{f_{0}}=\frac{2 \alpha}{1+2 \alpha}
$$

where $\alpha$ represents the total attenuation of the structure

$$
Q_{e_{1}}=Q_{e_{2}}=\frac{Q_{0}}{\alpha}
$$

Then the unloaded and loaded quality factors are linked through the following equation:

$$
Q_{0}=\frac{Q_{L}}{1+2 \alpha}
$$

The loaded quality factor is experimentally determined by the following expression:

$$
Q_{L}=\frac{f_{0}}{\Delta f}
$$

where $f_{0}$ is the resonant frequency and $\Delta f$ the $3 \mathrm{~dB}$ bandwidth.

We have chosen a silicon wafer featuring a $720 \mu \mathrm{m}$ thickness and we have performed 3D full wave electromagnetic simulations, which lead to the graph plotted in Figure 7 . We can

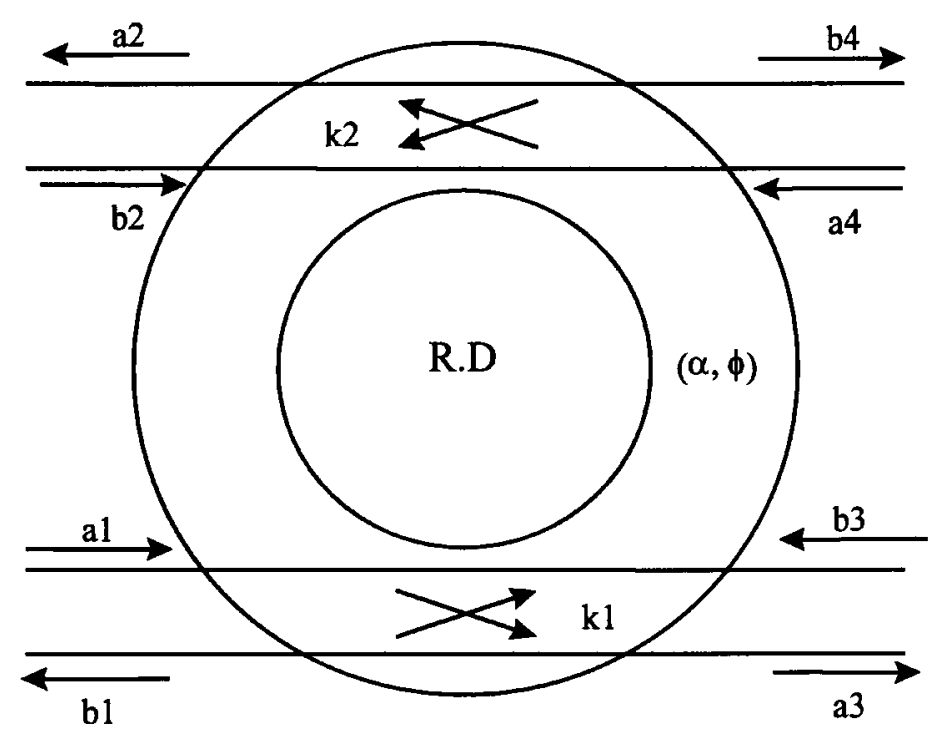

FIGURE 6 Typical schema showing the propagating way of the whispering gallery mode. 


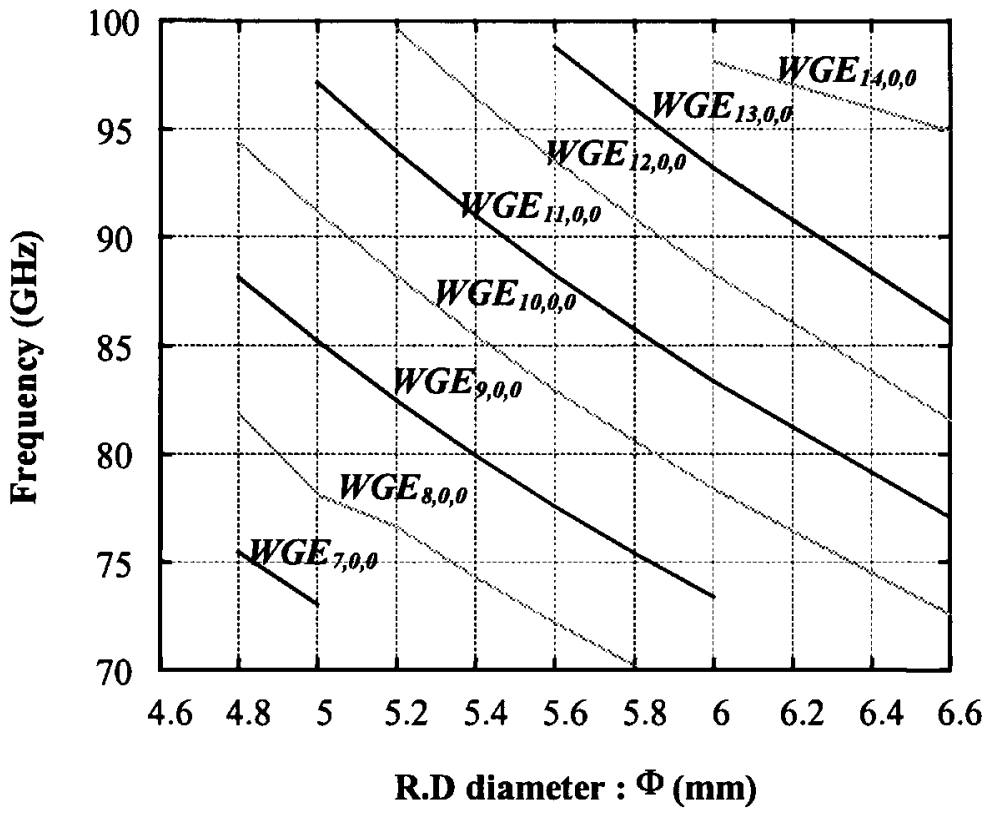

Modes $W_{G} E_{n, m}, 1$

FIGURE 7 Theoretical graph showing the WGE family modes versus the resonator diameter.

observed that a resonator's diameter of $6 \mathrm{~mm}$ leads to resonance frequency in the $95 \mathrm{GHz}$ range through a $\mathrm{WGE}_{13,0,0}$ mode excitation. The silicon resonator has been realized using a deep ion etching process. Finally, we have simulated the bend discontinuity in order to verify that no additional losses can be generated in it as reported in Figure 8.

The next section of this paper will be dedicated to the results obtained on the micromachined devices we designed.
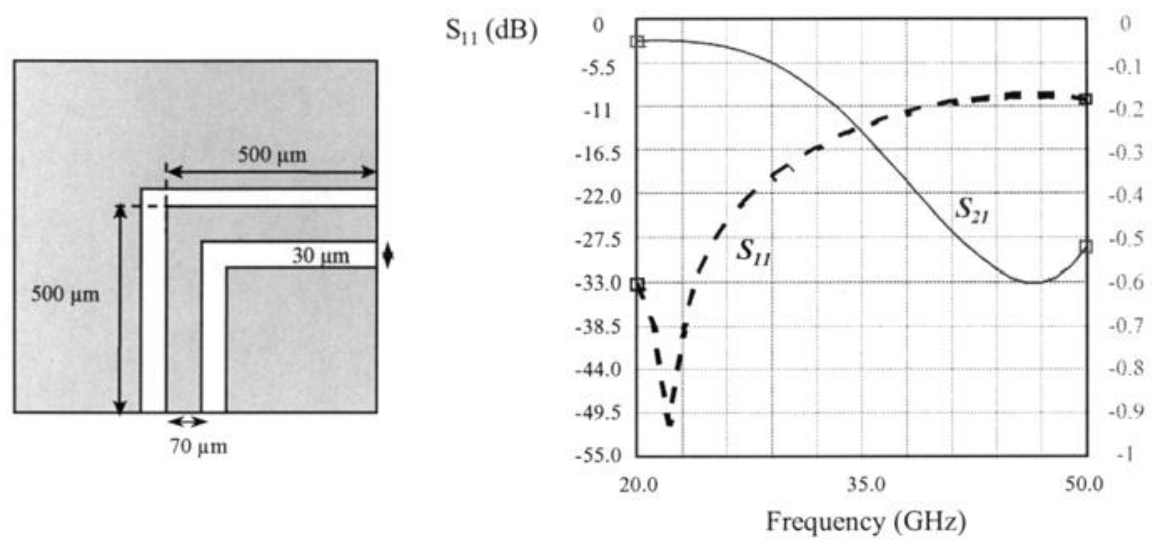

FIGURE 8 Schema and electromagnetic simulation of the bend discontinuity. 


\section{CHARACTERIZATION OF THE MICROMACHINED DEVICES}

The measurements have been done using a Wiltron VNA and SOLT calibration in the probe plane. We first measured the micromachined coplanar transmission line, which has been designed according to an optimal coupling with the resonator. In Figure 9 is shown the frequency evolution of the transmission coefficient of the CPW structure includ-

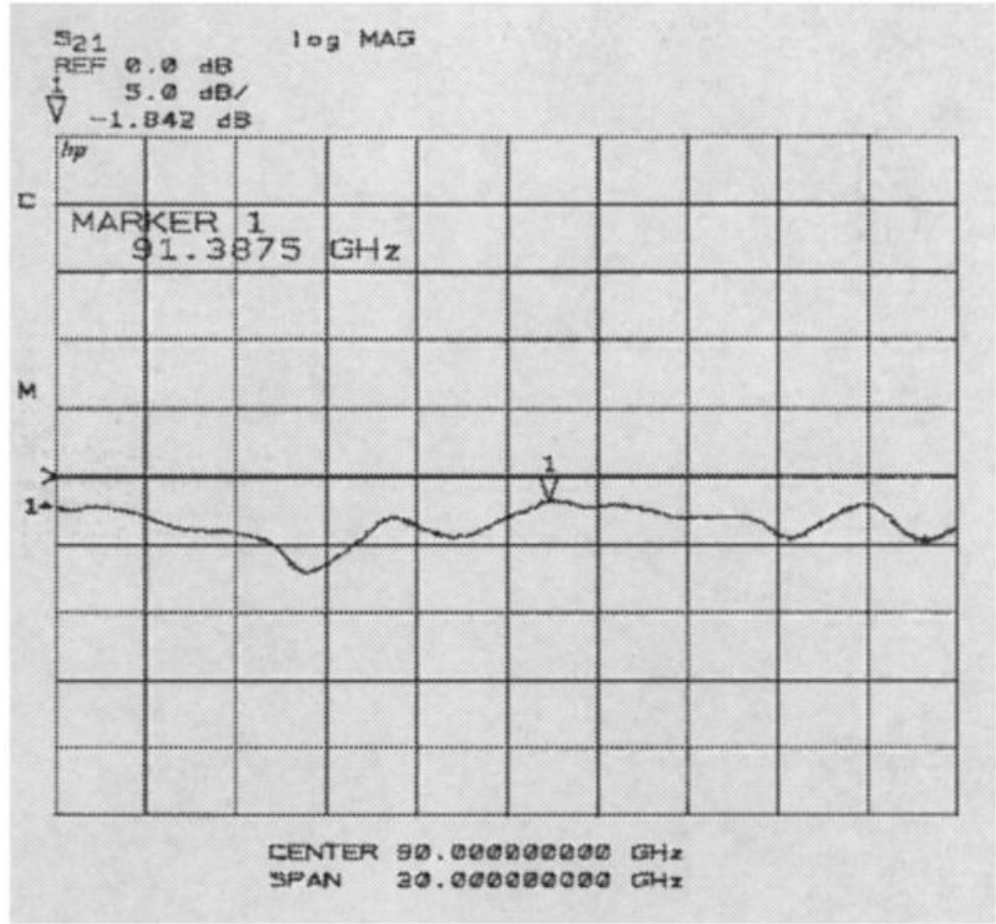

FIGURE 9 Frequency evolution of the transmission coefficient of the micromachined coplanar transmission line including the bend discontinuity between port I and III or II and IV.
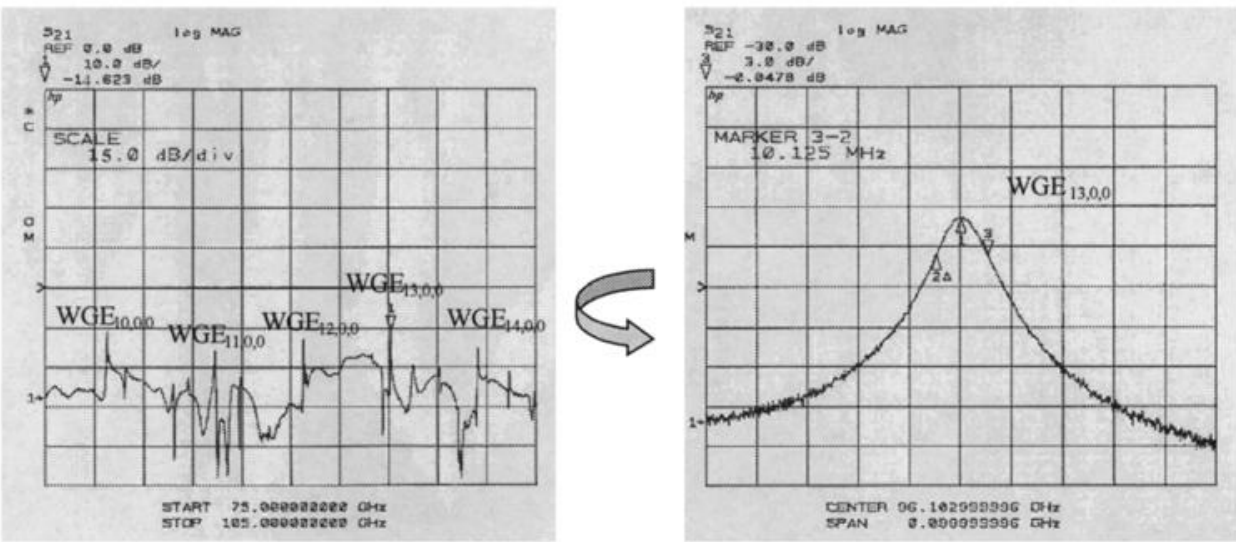

FIGURE 10 Measurements of the silicon micromachined cavity from $75 \mathrm{GHz}$ to $105 \mathrm{GHz}$ (each division represents $3 \mathrm{GHz}$ ) between ports I and II. Curve (b) measurements of the loaded quality factor according expression (6). 
ing the bend discontinuity and the bulk silicon access. We can observe a loss level in the $2 \mathrm{~dB}$ range up to $105 \mathrm{GHz}$, which confirms the good capabilities of the micromachined technology for the millimeter-wave interconnects. The cavity has been realized by placing the micromachined silicon resonator on a $510 \mu \mathrm{m}$ thick support. We present the frequency evolution of the transmission coefficient of the cavity between $75 \mathrm{GHz}$ and $105 \mathrm{GHz}$. We can observe the different WGE modes, which appear every $3 \mathrm{GHz}$. We have measured a quality factor of 9600 at $95 \mathrm{GHz}$ with an insertion loss level of $-15 \mathrm{~dB}$ which outperform all the results already published. Additional measurements have been done consisting in increasing the coupling between the resonator and the CPW structure and we have got quality factor of 2000 and insertion loss level of $-5 \mathrm{~dB}$ for a support thickness of $350 \mu \mathrm{m}$.

\section{CONCLUSIONS}

We present in this paper a micromachined technology allowing the realization of millimeter wave circuits on silicon featuring very high quality factor. Appropriate FEM simulations have been done to design micromachined devices like CPW transmission line and micromachined micro-cavity. Dielectric resonator acting on their whispering gallery modes feature larger dimensions than their counterpart acting on conventional TEM or hybrid modes which turn in too small size at millimeter-wave frequency. They can provide very high quality factor up to millimeter-wave. We have realized coplanar transmission line featuring transmission loss in the $2 \mathrm{~dB}$ range up to $100 \mathrm{GHz}$. The micromachined cavity we made consists in a coupling between coplanar wave guide and micromachined silicon dielectric resonator. We have got quality factor in the 10,000 range at $95 \mathrm{GHz}$. We demonstrated that silicon could be a promising material for millimeter-wave applications assuming appropriate technological process and accurate $3 \mathrm{D}$ full wave electromagnetic simulations.

\section{References}

[1] Weller, T. M., Katehi, L. P. B. and Rebeiz, G. R. (1995). High performance microshield line components. IEEE Transactions Theory and Techniques, 43(3), 534-543.

[2] Conedera, V., Fabre, N. and Dilhan, M. (1997). A simple optical system to optimize a high depth to width aspect ratio applied to a positive photoresist lithography process. Journal of Micromechanics and Microengineering, (7), 118-120.

[3] Willke, T. L. and Gearhart, S. S. (1996). Novel micromachined Liga micromachined transmission lines and filters. IEEE MTT-S Digest.

[4] Rogner, A., Eichert, J., Munchmeyer, D., Peters, R. P. and Mohr, J. (1992). The LIGA technique. What are the new opportunities. Journal of Micromechanics and Microengineering, (2).

[5] Saint-Etienne, E., Pons, P., Blasquez, G., Temple, P., Conedera, V., Dihlan, M., Chauffleur, X., Menini, Ph., Plana, R., Parra, T., Guillon, B. and Lalaurie, J. C. (1998). A dedicated micromachining technology for highaspect ratio millimetre-wave circuits. Sensors and Actuators, A 68, 435-441.

[6] Guillon, B., Cros, D., Parra, T., Pons, P., Lalaurie, J. C., Plana, R. and Graffeuil, J. (1998). Si micromachining and whispering gallery dielectric resonator modes. An original issue for high Q coplanar millimeter-wave cavity. Proceedings of 4th International Conference on Millimeter and Submillimeter Waves and Applications, International society for optical engineering, San Diego, July 20-23 1998, pp. 120-129.

[7] Reyes, A. C., El-Ghazaby, S. M., Dorn, S., Dydyk, M., Schroder, D. K. and Patterson, H. (1995). Coplanar waveguides and microwave inductors on silicon substrates. IEEE Transactions on Microwave and Technique, 43(9), 2016-2022.

[8] Wu, Y., Yang, S., Gamble, H. S., Armstrong, B., Fusco, V. and Stewart, J. (1999). SiO2 interface layer effects on microwave loss of high resistivity CPW line. IEEE Microwave and Guided Wave Letters, 9(1), 10-12.

[9] Ponchak, G. (1999). RF transmission lines on silicon substrates. Proc of 29th European Microwave Conference, Munich 1999, pp. 158-161. 
[10] Gamble, H. S., Armstrong, B. M., Mitchell, S. J. N., Wu, Y., Fusco, V. and Stewart, J. (1999). Low loss CPW lines on surface stabilized high-resistivity silicon. IEEE Microwave and Guided Wave Letters, 9(10), 395-397.

[11] Blondy, P., Brown, A. R., Cros, D. and Rebeiz, G. M. (1998). Low loss micromachined filters for millimeterwave telecommunications systems. Proc of MTT-Symp, Vol. 3, pp. 1181-1184.

[12] Papapolymerou, J., Cheng, J. C., East, J. and Katehi, L. P. B. (1997). A micromachined high Q X-band resonator. IEEE Microwave and Guided Wave Letters, 7(6).

[13] Cros, D. and Guillon, P. (1990). Whispering gallery dielectric resonator modes for W-band devices. IEEE Transactions on Microwave Theory and Techniques, 38(11), 1667-1674. 

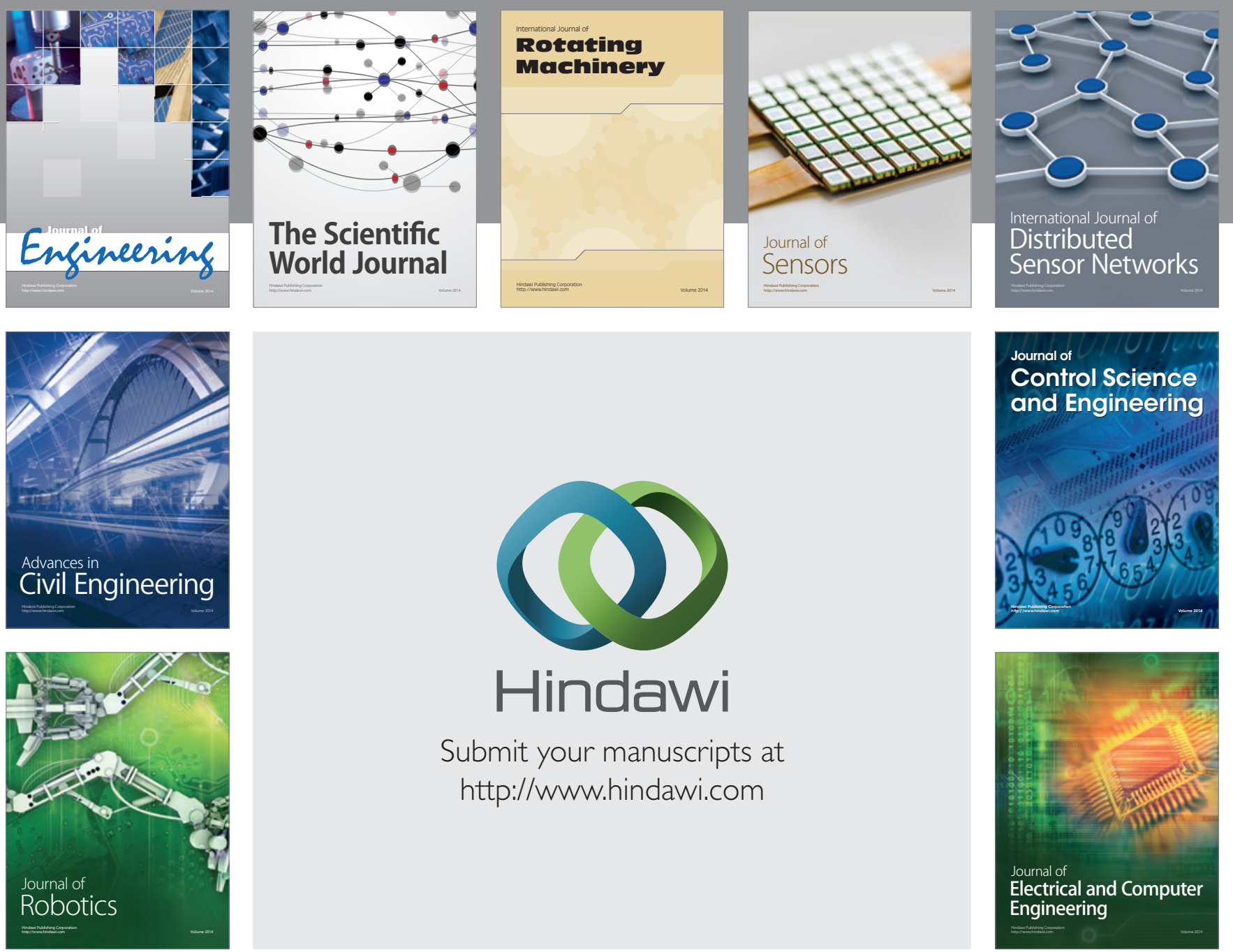

Submit your manuscripts at

http://www.hindawi.com
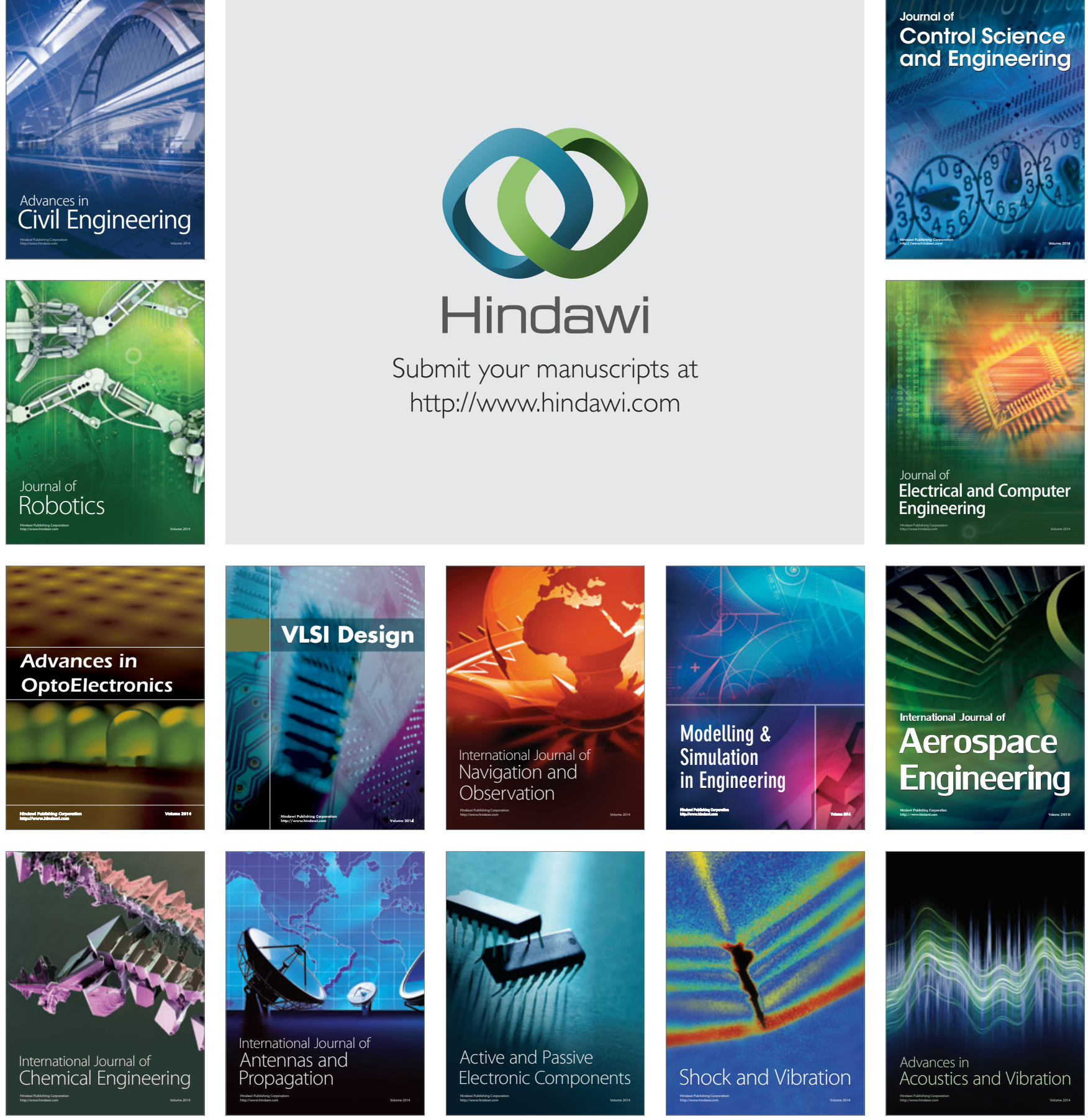